\title{
Cardiogenic Shock Caused by a Large Sub-Epicardial Hematoma Complicating Percutaneous Coronary Intervention
}

\author{
Yoshiharu Higuchi*, Daisuke Fukamachi, Nobuyuki Fujii, Toshihiko Nishida, \\ Atsushi Hirayama \\ Division of Cardiology, Department of Medicine, Nihon University School of Medicine, Tokyo, Japan \\ Email: ${ }^{*}$ yhiguchi-ja@umin.net
}

Received 24 May 2015; accepted 12 June 2015; published 15 June 2015

Copyright (C) 2015 by authors and Scientific Research Publishing Inc.

This work is licensed under the Creative Commons Attribution International License (CC BY).

http://creativecommons.org/licenses/by/4.0/

\section{(c) (i) Open Access}

\section{Abstract}

A 56-year-old man with a history of old myocardial infarction and coronary artery bypass grafting was performed percutaneous coronary intervention to the totally occluded lesion of native left anterior descending artery. He developed a coronary arterial perforation that led to a large dissecting sub-epicardial hematoma. Emergent surgery was performed, and a large hematoma was removed from the sub-epicardial space. Continuous drainage of blood was observed after surgery, and the patient died due to the development of multiorgan dysfunction. An autopsy revealed an extensive sub-epicardial hematoma on the lateral wall of the left ventricle and broad necrosis of the myocardium in the lateral wall.

\section{Keywords}

Sub-Epicardial Hematoma, Cardiac Tamponade, Percutaneous Coronary Intervention, Coronary Artery Bypass Grafting

\section{Introduction}

With an incidence of less than $0.5 \%$, coronary perforation is not a frequently occurring complication of percutaneous coronary intervention (PCI) [1]. This complication is seen more frequently in the treatment of chronic total occlusion [1]. However, cardiac tamponade following coronary perforation has a poor prognosis [2]. Cardiac tamponade is generally attributed to extravasation of blood into the pericardial space. A relatively small blood volume within the pericardial space may cause severe hemodynamic instability and death, even with early diagnosis and prompt treatment. While pericardiocentesis is often performed to stabilize hemodynamic state, almost

\footnotetext{
${ }^{*}$ Corresponding author.
}

How to cite this paper: Higuchi, Y., Fukamachi, D., Fujii, N., Nishida, T. and Hirayama, A. (2015) Cardiogenic Shock Caused by a Large Sub-Epicardial Hematoma Complicating Percutaneous Coronary Intervention. Case Reports in Clinical Medicine, 4, 233-236. http://dx.doi.org/10.4236/crcm.2015.46045 
$40 \%$ of the patients require further emergency surgical intervention [2] [3]. Another type of cardiac tamponade, which is caused by pooling of blood in the epicardial space, is reported [4]-[7]. The "sub-epicardial hematoma" is rare, but develops rapidly toward circulatory collapse. We herein report a large dissecting sub-epicardial hematoma which resulted in cardiogenic shock.

\section{Case Presentation}

The patient was a 56-year-old man with dyslipidemia and a long history of coronary artery disease. He presented with recurrent angina and dyspnea on effort 2 months prior to admission. His medical history included anterior myocardial infarction at the age of 37 years and coronary artery bypass grafting (CABG) with grafting of the left internal thoracic artery (LITA) to the second diagonal branch artery and a saphenous vein graft (SVG) to the left anterior descending (LAD) coronary artery at the age of 39 years. He was admitted to our hospital, and cardiac catheterization was performed. Coronary angiography showed a chronic total occlusion (CTO) in the native LAD and occlusion of the SVG (Figure 1(a)). His LITA was patent and blood flowed well to the second diagonal branch, but the distal portion of the native LAD was not perfused (Figure $\mathbf{1}(\mathbf{b})$ ). A coronary intervention to treat the CTO lesion was performed 1 week later. To obtain further backup force, a floppy 0.014-inch guidewire was crossed to the first diagonal branch, and a 2.0- $\times 12.0-\mathrm{mm}$ balloon was inflated as an anchoring balloon. A stiff guide wire was passed to the second diagonal branch, but it could not be passed to the distal LAD (Figure 1(c)). A coronary angiogram showed pooling of the contrast medium along the first diagonal branch (Figure 1(d)). Extravasation of blood was observed a few minutes later, and the patient developed cardiogenic shock (Figure 1(e)). We attempted to evacuate the blood surrounding the left ventricle by percutaneous pericardiocentesis; however, the hematoma was localized on the lateral wall of the heart, and a puncture needle could not reach the hematoma. Therefore, emergent surgery was performed to remove the hematoma by median sternotomy. There was no blood or clot in the pericardial space, and the lateral epicardial surface of the left ventricle was swollen and dark red (Figure 2(a)). The hematoma may have been caused by dissection of the epicardium from the myocardium. Upon incising the epicardium, a large sub-epicardial hematoma was evacuated. The patient required percutaneous cardiopulmonary support to maintain the systemic circulation after surgery. Continuous drainage of blood from the pericardial space was observed, and echocardiography revealed a large hematoma on the lateral wall of the left ventricle (Figure 2(b)). Invasive thoracotomy for removal of the hematoma could not be performed because multiorgan dysfunction developed. The patient died 3 weeks after surgery. An autopsy was carried out, and examination of the heart revealed an extensive sub-epicardial hematoma measuring $4 \times 7 \times 8 \mathrm{~cm}$ on the lateral wall of the left ventricle (Figure 2(c), Figure 2(d)). Broad necrosis of the myocardium in the lateral wall was observed.

\section{Discussion}

We have reported a rare complication of PCI that resulted in cardiac tamponade due to a sub-epicardial hematoma. An autopsy revealed broad infarction of the myocardium in the lateral wall. Myocardial infarction probably developed secondary to compression of the myocardium and diagonal arteries by the hematoma.

Several cases of sub-epicardial hematoma following PCI were previously reported [4] [6] [7]. Fukushima et al. reported a sub-epicardial hematoma during PCI to the lesion of the obtuse marginal branch, and the patient had a

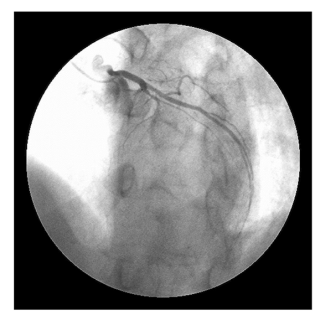

(a)

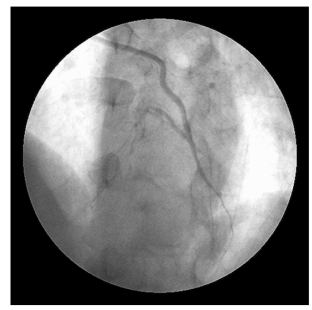

(b)

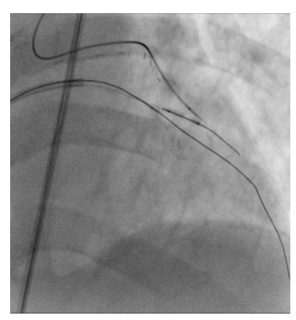

(c)

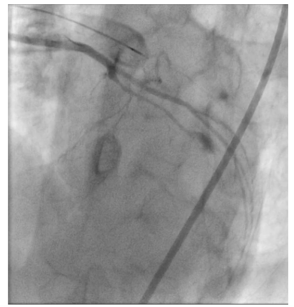

(d)

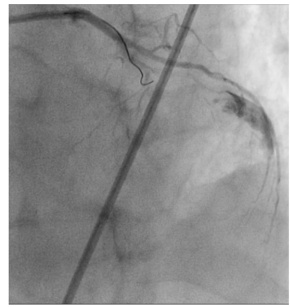

(e)

Figure 1. Coronary angiography and intervention. (a) Coronary angiography shows chronic total occlusion of left anterior descending artery; (b) Bypass angiography shows left internal thoracic artery graft to the second diagonal branch; (c) The anchor balloon is inflated in the first diagonal branch, and a stiff wire is crossing to the second diagonal branch; (d) Pooling of contrast medium is present in the extravascular area; (e) Contrast medium is extravasated into the sub-epicardial space. 


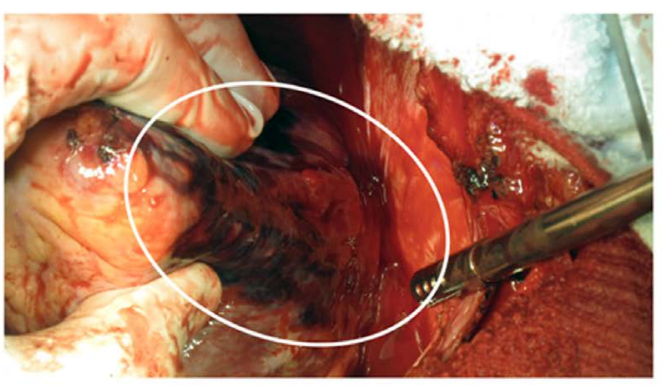

(a)

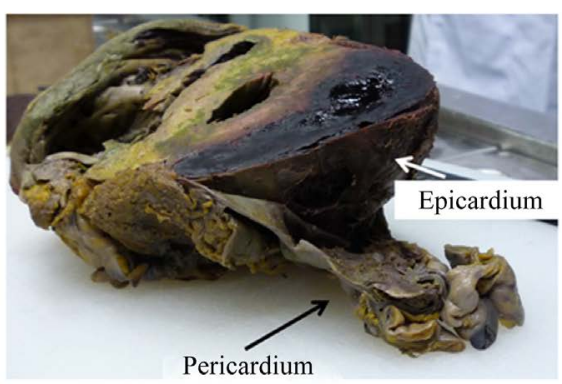

(c)

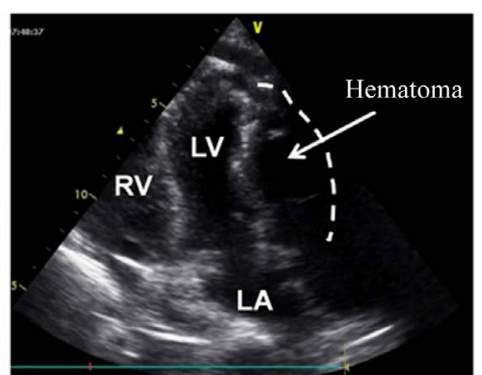

(b)

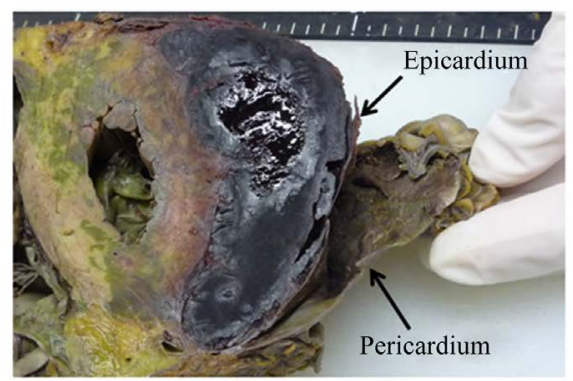

(d)

Figure 2. Sub-epicardial hematoma. (a) Lateral wall of the heart after incision of the pericardium. A large sub-epicardial hematoma is indicated with the white circle; (b) Echocardiography shows a huge low-echoic mass besides the lateral wall of the left ventricle; (c) and (d) The autopsy specimen shows an extensive sub-epicardial hematoma on the lateral wall of the left ventricle. There is no clot in the pericardial space, and a large hematoma is present between the epicardium and the myocardium (sub-epicardial space).

history of CABG [4]. Shekar et al. also reported a similar case in patient with a history of CABG during a cutting balloon angioplasty to the SVG [6]. Kashiwase et al. reported a dissecting sub-epicardial hematoma, but the patient had no history of previous cardiac surgery [7]. The patient had a past history of collagen disease, and the authors suggested that connective tissue disorder might weaken the binding power between the epicardium and the myocardium.

We performed PCI to treat a CTO lesion of the native coronary artery in a patient with a history of previous CABG. We are not sure whether a floppy guidewire or a balloon injured the first diagonal branch. Since the pooling of blood was observed at the distal portion of the vessel, we conjecture that a floppy guidewire penetrated the intima of the vessel and advanced into the sub-epicardial space. A meta-analysis of the risk of complications during CTO coronary intervention demonstrated that coronary perforation is not a rare complication and has an incidence rate of $2.9 \%$, but that cardiac tamponade and death are rare with incidence rates of $0.3 \%$ and $0.2 \%$, respectively [8]. Cardiac tamponade is generally caused by pooling of blood in the pericardial space between the epicardium and pericardium. In contrast, sub-epicardial hematomas are caused by dissection of the epicardium from the myocardium and develops rapidly; i.e., they are self-propagating [6]. Cardiac surgery including CABG may cause postoperative pericardial adhesion. It is possible that the adhesion of the pericardial space caused by the previous CABG contributed to the progression of the dissecting sub-epicardial hematoma.

\section{Conclusion}

We reported a large dissecting sub-epicardial hematoma, a rare complication of PCI. A dissection of the subepicardial space developed rapidly and a large hematoma on the lateral wall of the left ventricle caused cardiac tamponade. We propose that sub-epicardial dissection should be recognized as one of the most serious complications of coronary intervention in patients with a history of previous cardiac surgery.

\section{Competing Interests}

There are non-financial competing interests. 


\section{References}

[1] Gunning, M.G., Williams, I.L., Jewitt, D.E., Shah, A.M., Wainwright, R.J. and Thomas, M.R. (2002) Coronary Artery Perforation during Percutaneous Intervention: Incidence and Outcome. Heart, 88, 495-498. http://dx.doi.org/10.1136/heart.88.5.495

[2] Javaid, A., Buch, A.N., Satler, L.F., Kent, K.M., Suddath, W.O., Lindsay Jr., J., Pichard, A.D. and Waksman, R. (2006) Management and Outcomes of Coronary Artery Perforation during Percutaneous Coronary Intervention. The American Journal of Cardiology, 98, 911-914. http://dx.doi.org/10.1016/j.amjcard.2006.04.032

[3] Fejka, M., Dixon, S.R., Safian, R.D., O’Neill, W.W., Grines, C.L., Finta, B., Marcovitz, P.A. and Kahn, J.K. (2002) Diagnosis, Management, and Clinical Outcome of Cardiac Tamponade Complicating Percutaneous Coronary Intervention. The American Journal of Cardiology, 90, 1183-1186. http://dx.doi.org/10.1016/S0002-9149(02)02831-X

[4] Furushima, H., Matsubara, T., Tamura, Y., Yamazoe, M., Konno, T., Ida, T., Aizawa, Y., Moro, H. and Watanabe, H. (1997) Coronary Artery Perforation with Subepicardial Hematoma. Catheterization and Cardiovascular Diagnosis, 41, 59-61. http://dx.doi.org/10.1002/(SICI)1097-0304(199705)41:1<59::AID-CCD15>3.0.CO;2-L

[5] Misfeld, M., Khan, S.A., Ilsley, C. and Amrani, M. (2002) Epicardial Haematoma: Rare Cause of Acute Myocardial Ischaemia. European Journal of Cardio-Thoracic Surgery, 21, 119-120. http://dx.doi.org/10.1016/S1010-7940(01)01071-5

[6] Shekar, P.S., Stone, J.R. and Couper, G.S. (2004) Dissecting Sub-Epicardial Hematoma—Challenges to Surgical Management. European Journal of Cardiothorac Surgery, 26, 850-853. http://dx.doi.org/10.1016/j.ejcts.2004.06.022

[7] Kashiwase, K., Ueda, Y., Ogasawara, N., Oyabu, J., Okada, K., Murakawa, T., Hirayama, A. and Kodama, K. (2008) A Large Dissecting Sub-Epicardial Hematoma and Cardiac Tamponade Following Elective Percutaneous Coronary Intervention. Journal of Cardiology, 52, 163-166. http://dx.doi.org/10.1016/j.jicc.2008.05.006

[8] Patel, V.G., Brayton, K.M., Tamayo, A., Mogabgab, O., Michael, T.T., Lo, N., Alomar, M., Shorrock, D., Cipher, D., Abdullah, S., Banerjee, S. and Brilakis, E.S. (2013) Angiographic Success and Procedural Complications in Patients Undergoing Percutaneous Coronary Chronic Total Occlusion Interventions: A Weighted Meta-Analysis of 18,061 Patients from 65 Studies. Journal of American Courage of Cardiology: Cardiovascular Interventions, 6, 128-136. http://dx.doi.org/10.1016/j.jcin.2012.10.011 